\title{
Effects of Lactobacillus on hyperlipidemia in high-fat diet-induced mouse model
}

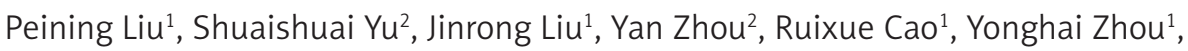 \\ Linwei Shi ${ }^{1}$, Jimei $\mathrm{Du}^{2}$
}

\begin{abstract}
${ }^{1}$ The Second Affiliated Hospital \& Yuying Children's Hospital of Wenzhou Medical University, Wenzhou, Zhejiang, China

${ }^{2}$ Wenzhou Key Laboratory of Sanitary Microbiology, School of Laboratory Medicine and Life Sciences, Wenzhou Medical University, Wenzhou, Zhejiang, China
\end{abstract}

Submitted: 15 May 2019

Accepted: 12 October 2019

Arch Med Sci

DOI: https://doi.org/10.5114/aoms.2020.98927

Copyright $\odot 2020$ Termedia \& Banach

\begin{abstract}
Introduction: Hyperlipidemia refers to a group of lipid metabolism disorders characterized by increased levels of total cholesterol, triglyceride, and/or lowdensity lipoprotein cholesterol and/or decreased levels of high-density lipoprotein cholesterol. This study aims to investigate the effects of Lactobacillus on lipid metabolism and hepatic steatosis in male mice fed with a high-fat diet by measuring blood lipid, hepatic function and hepatocyte morphology. Material and methods: Eighty male Institute of Cancer Research (ICR) mice were fed with a high-fat diet for six weeks to establish hyperlipidemic models. Then, mice were treated with a high or low concentration of Lactobacillus of human source, mouse source, or plant source, respectively.

Results: After three weeks of therapy, except for the human Lactobacillus treatment group, the blood cholesterol, triglyceride and low-density lipoprotein cholesterol in mice treated with Lactobacillus of mouse and plant source were lower than those in the hyperlipidemic model group. After four weeks of treatment, the levels of blood biochemical indexes in mice in all treatment groups were significantly different, when compared to those in the hyperlipidemic model group.

Conclusions: Lactobacillus may regulate blood lipid in mice fed with a highfat diet. Lactobacillus can improve the high cholesterol, high blood lipid, and injury of hepatic function, and prevent further development of atherosclerosis caused by a high-fat diet to some extent. Correct dietary structure is the basis for the treatment of dietary hyperlipidemia and its complications.
\end{abstract}

Key words: Lactobacillus, hyperlipidemia, ICR mice, high-fat diet.

\section{Introduction}

Hyperlipidemia refers to a group of lipid metabolism disorders characterized by increased levels of total cholesterol (TC), triglyceride (TG), and/or low-density lipoprotein cholesterol (LDL-C) and/or decreased levels of high-density lipoprotein cholesterol (HDL-C). Hyperlipidemia may promote the occurrence and development of atherosclerosis, and become an important risk factor for cardiovascular and cerebrovascular diseases, thereby seriously threatening human health [1].

Intestinal microflora plays a key role in maintaining human health. Lactobacillus is an important component of normal intestinal microflora,

\author{
Corresponding author: \\ Jimei Du \\ Wenzhou Key Laboratory \\ of Sanitary Microbiology \\ School of Laboratory \\ Medicine and Life Sciences \\ Wenzhou Medical University \\ Chashan University Town \\ Wenzhou Medical University \\ Teaching Building 7A323 \\ c 325035China \\ E-mail: dujimei311@163.com
}


and has many bioactivities and effects. Studies have demonstrated that Lactobacillus is negatively correlated with lipid levels and blood glucose [2]. The possible mechanism is that Lactobacillus may convert cholesterol into fecal sterol by oxidization in the intestine, which can no longer be returned to the liver through the intestinal-hepatic circulation, thereby lowering cholesterol levels. The special enzymes produced by Lactobacillus also have an effect of lowering cholesterol synthesis. 3-hydroxy-3-methylglutaric coenzyme A reductase (HMGCR) is an important enzyme in cholesterol synthesis (a rate-limiting enzyme for cholesterol synthesis). The study conducted by Chen et al. confirmed that Lactobacillus could inhibit the HMGCR level in human hepatoma Hepg2 cells through experiments, which is another theoretical basis for the anti-hyperlipidemic effect of Lactobacillus [3]. In addition, Lactobacillus may adhere to and colonize the intestinal mucosa, which absorbs part of the cholesterol, converts it into bile salts and excretes this from the body. Hence, it can significantly reduce the intestinal absorption of cholesterol [4]. The study conducted by Zavišić et al. has proven the effect of Lactobacillus in reducing cholesterol and triglyceride in mice, and the safety of treatment [5]. This study aims to investigate the effects of Lactobacillus on lipid metabolism and hepatic steatosis in male mice fed with a high-fat diet by measuring blood lipid, hepatic function and hepatocyte morphology.

\section{Material and methods}

\section{Source of strains}

Lactobacillus strains were preserved in the laboratory, and were isolated from healthy human feces, Institute of Cancer Research (ICR) male mouse feces, and a brand of yogurt. These strains were screened through cholesterol degradation experiments.

\section{Reagents and medium}

Xuezhikang (a partially purified extract of red yeast Chinese rice with multiple components that was used for the treatment of cardiovascular and cerebrovascular diseases caused by hyperlipidemia and atherosclerosis) was purchased from the Beijing WBL Peking University Biotech Co., Ltd (WPU) (Beijing, People's Republic of China). Normal diet and hyperlipidemic diet were provided by Shanghai Pluton Biotechnology Co., Ltd. The cholesterol, bile salt, ferric chloride, ammonium ferric sulfate, De Man-Rogosa-Sharpe (MRS) agar medium, eosin methylene blue medium, and MRS broth medium were provided by the microbiology laboratory of our institution. The simulated gastric juice, cholesterol medium and bile salt medium were prepared according to the references $[6,7]$.

\section{Main instruments and apparatus}

Vertical pressure steam sterilizer, BXM-30R; water-isolated thermostat incubator, DRP-9080; transmission electron microscope, Hitachi H750O.

\section{Animals}

One hundred male ICR mice, weighing 30-35 g, were provided by the experimental animal center of our institution.

\section{Experiment methods}

\section{Strains}

Lactobacillus strains were isolated from healthy human feces, ICR male rat feces, and a brand of yoghurt by MRS medium. The ability of each strain to degrade cholesterol in vitro [8] and the ability of Lactobacillus to tolerate bile salt and acid were determined using the methods mentioned in the literature to screen out the suitable strains.

\section{Preparation of bacterial solution}

The appropriate amount of identified Lactobacillus was scraped, and the corresponding bacterial solution was prepared by Maxwell turbidimetry.

\section{Establishment of the hyperlipidemia mouse} model

Ninety male mice were randomly divided into different groups, with 10 mice in each group. Mice in group A-H were fed with a high-fat diet [9], while mice in group I were fed with a normal diet.

\section{Treatment}

The male mice that were successfully established into models were randomly divided into 10 groups, with nine mice in group $A-G$ and seven mice in group $\mathrm{H}-$ J. Mice in group $\mathrm{A}$ were treated with low concentrations of human Lactobacillus (human low group), mice in group B were treated with high concentrations of human Lactobacillus (human high group), mice in group $C$ were treated with low concentrations of mouse Lactobacillus (mouse low group), mice in group D were treated with high concentrations of mouse Lactobacillus (mouse high group), mice in group E were treated with low concentrations of plant Lactobacillus (plant low group), and mice in group $\mathrm{F}$ were treated with high concentrations of plant Lactobacillus (plant high group). Furthermore, mice in group $G$ were treated with Xuezhikang, mice in group $\mathrm{H}$ were treated with a high-fat diet, and mice in group I were treated with a normal diet (diet group), while mice in group J were set as a control, and treated with a normal diet. Mice in the treatment group (group A-F) were fed with a high-fat diet, instead of a normal diet, 
after the successful establishment of the hyperlipidemic model. At the same time, each mouse was given $0.1 \mathrm{ml}$ of bacterial solution, daily. In the low concentration groups, the amount of bacteria was $3 \times 10^{9} \mathrm{CFU} / \mathrm{ml}$, while in the high concentration groups, the amount of bacteria was $3 \times 10^{10} \mathrm{CFU} / \mathrm{ml}$. Furthermore, $0.1 \mathrm{ml}(24 \mathrm{mg} / \mathrm{ml})$ of Xuezhikang was given daily by regular intragastric administration to each mouse treated with Xuezhikang (group G) [10] for four weeks. Mice in the normal diet treatment group were switched to a normal diet after successful model establishment, and each mouse was given $0.1 \mathrm{ml}$ of saline regularly, daily. Mice in the hyperlipidemic model group and normal control group were continuously fed, and each mouse was given $0.1 \mathrm{ml}$ of saline regularly, daily. The body weights of mice were observed and measured daily.

\section{Results of treatment}

Obesity index and visceral volume ratio: Body weight, body length and body weight before execution were measured daily, and the obesity index was calculated. The liver, spleen, kidney and other organs were weighed, and the liver and spleen index were calculated after these mice were executed.

Intestinal flora assay: The feces of mice were taken using the forcing method every week, and the number of Enterococcus, Enterobacter, Lactobacillus and total bacteria were determined.

Serological detection: Blood was collected from the eyeball of mice, and serum was separated. Then, the serological indicators (TC, TG, HDL-C, LDL-C, AST(U/L), ALT(U/L) and $\mathrm{Al}(\mathrm{TC} / \mathrm{HDL}-\mathrm{C})$ ) were determined using an automatic blood biochemical analyzer.

Electronic microscopic observation: The liver was immediately collected after mice were sacrificed, and the structure of the liver was observed under an electron microscope.

\section{Statistic analysis}

The data obtained in the present study were collected, and the statistics and calculations in the early stage were performed in Microsoft Excel 2010. Variance analysis and correlation analysis were carried out using the SPSS 18.0 software (Chinese version).

\section{Results}

\section{Screening of Lactobacillus}

Three strains of Lactobacillus that met the requirements were screened from healthy human, ICR mice and yogurt using the cholesterol degradation test, and acid and bile salt tolerance test: the code of Lactobacillus $\mathrm{H}$ of human source, Lactobacillus I of mouse source, and Lactobacillus Z of plant source was number 1, 2 and 3 (Table I).

\section{Establishment of the hyperlipidemic mouse model}

After high-fat diet feeding, the obesity index of mice in the normal control group and hyperlipidemic model group was $15.68 \pm 0.44$ and 16.25 \pm 0.55 , respectively, at the end of the sixth week. Hence, the model was successfully established.

\section{Serum biochemical indicators after three weeks of treatment}

The results of the serum biochemical indicators of mice in each group after three weeks of treatment are presented in Table II.

In the human low group, TG was significantly lower compared to the hyperlipidemic group $(p<0.01)$, while LDL-C was significantly lower compared to the control group $(p<0.05)$. In the human high group, the levels of TC, TG and $\mathrm{HDL}-\mathrm{C}$ were lower than those in the hyperlipidemic group $(p<0.05)$, while the levels of LDL-C and HDL-C were lower than those in the human low group $(p<0.05)$. In the mouse low group, the levels of TC, TG, HDL-C and LDL-C were all lower than those in the hyperlipidemic group $(p<0.05)$. In the mouse high group, the levels of TC, TG, HDL-C and LDL-C were all lower than those in the hyperlipidemic group $(p<0.05)$, and the difference in level of TG was statistically significant compared to the mouse low group $(p<0.05)$. In the plant low group, the levels of TC, TG, HDL-C and LDL-C were all lower than those in the hyperlipidemic group $(p<0.05)$, while the level of TC was lower than that in the control group ( $p<0.05$ ). The levels of TC, TG, HDL-C and LDL-C were lower in the plant high group than

Table I. Lactobacillus screening results

\begin{tabular}{|lccccc|}
\hline $\begin{array}{l}\text { Project/ } \\
\text { groups }\end{array}$ & $\begin{array}{c}\text { Degrading rate } \\
\text { of cholesterol (\%) }\end{array}$ & $\begin{array}{c}\text { Acid resistant rate } \\
(\%) \\
(\mathrm{pH}=3)\end{array}$ & $\begin{array}{c}\text { Bile salt tolerance } \\
\text { rate (\%) } \\
(0.1 \%)\end{array}$ & $\begin{array}{c}\text { Bile salt tolerance } \\
\text { rate (\%) } \\
(0.3 \%)\end{array}$ & $\begin{array}{c}\text { Bile salt tolerance } \\
\text { rate }(\%) \\
(0.5 \%)\end{array}$ \\
\hline No. 1 & 61.41 & 72.76 & 66.4 & 14.5 & 7.0 \\
\hline No. 2 & 54.31 & 85.5 & 38.9 & 14.1 & 6.2 \\
\hline No. 3 & 41.20 & 81.2 & 52.5 & 14.8 & 7.1 \\
\hline
\end{tabular}

No. 1 is Lactobacillus of human source, No. 2 is Lactobacillus of mouse source, No. 3 is Lactobacillus of plant source. 
Table II. Three weeks of treatment, blood biochemical index results $(\bar{x} \pm s, n=4)$

\begin{tabular}{|c|c|c|c|c|c|c|c|}
\hline $\begin{array}{l}\text { Project/ } \\
\text { groups }\end{array}$ & $\begin{array}{c}\mathrm{TC} \\
(\mathrm{mmol} / \mathrm{l})\end{array}$ & $\begin{array}{c}\mathrm{TG} \\
(\mathrm{mmol} / \mathrm{l})\end{array}$ & $\begin{array}{c}\text { HDL-C } \\
(\mathrm{mmol} / \mathrm{l})\end{array}$ & $\begin{array}{c}\text { LDL-C } \\
(\mathrm{mmol} / \mathrm{l})\end{array}$ & $\begin{array}{l}\text { AST } \\
(U / I)\end{array}$ & $\begin{array}{c}\text { ALT } \\
(\mathrm{U} / \mathrm{l})\end{array}$ & Al \\
\hline Group A & $3.48 \pm 0.76$ & $0.93 \pm 0.50^{\Delta}$ & $0.79 \pm 0.12$ & $0.43 \pm 0.09^{\#}$ & $101.00 \pm 21.56$ & $89.50 \pm 23.69$ & $3.41 \pm 0.43$ \\
\hline Group B & $3.04 \pm 0.34^{*}$ & $1.62 \pm 0.35^{\Delta}$ & $0.59 \pm 0.07^{\& *}$ & $0.28 \pm 0.08^{\&}$ & $88.00 \pm 22.49$ & $81.50 \pm 9.43$ & $4.15 \pm 0.51$ \\
\hline Group C & $2.17 \pm 0.70^{\star}$ & $1.81 \pm 0.23^{\Delta}$ & $0.48 \pm 0.05^{\star \Delta}$ & $0.21 \pm 0.05^{\star \Delta}$ & $95.40 \pm 25.21$ & $78.00 \pm 32.65$ & $4.06 \pm 0.46$ \\
\hline Group D & $2.09 \pm 0.86^{\Delta}$ & $1.21 \pm 0.06^{\& \Delta}$ & $0.43 \pm 0.16^{\star}$ & $0.18 \pm 0.10^{*}$ & $90.33 \pm 13.58$ & $103.33 \pm 19.22$ & $3.80 \pm 0.25$ \\
\hline Group E & $2.34 \pm 0.37^{\Delta \#}$ & $1.33 \pm 0.66^{\Delta}$ & $0.49 \pm 0.03^{\Delta}$ & $0.27 \pm 0.04^{\star}$ & $103.25 \pm 32.87$ & $82.50 \pm 34.10$ & $4.21 \pm 0.70$ \\
\hline Group F & $2.66 \pm 0.15^{\Delta \#}$ & $1.52 \pm 0.60^{\Delta}$ & $0.49 \pm 0.07^{\star}$ & $0.23 \pm 0.02^{\Delta}$ & $117.75 \pm 33.29$ & $106.50 \pm 24.08$ & $4.51 \pm 0.86$ \\
\hline Group G & $2.31 \pm 0.25^{\#}$ & $1.42 \pm 0.29^{\Delta}$ & $0.44 \pm 0.04^{\Delta}$ & $0.18 \pm 0.04^{\Delta}$ & $78.50 \pm 9.15$ & $83.50 \pm 11.12$ & $4.24 \pm 0.26$ \\
\hline Group H & $3.60 \pm 0.30$ & $1.19 \pm 0.20^{\Delta}$ & $0.66 \pm 0.06$ & $0.30 \pm 0.02$ & $123.00 \pm 57.98$ & $121.00 \pm 55.15$ & $4.49 \pm 0.07$ \\
\hline Group I & $3.68 \pm 0.17$ & $4.50 \pm 0.52$ & $0.81 \pm 0.15$ & $0.39 \pm 0.05$ & $99.00 \pm 21.79$ & $121.00 \pm 1.00$ & $4.07 \pm 0.98$ \\
\hline Group J & $3.50 \pm 0.55$ & $1.23 \pm 0.47^{\Delta}$ & $0.62 \pm 0.15$ & $0.21 \pm 0.05^{\star}$ & $78.50 \pm 21.51$ & $155.00 \pm 38.18$ & $4.75 \pm 0.49$ \\
\hline
\end{tabular}

${ }^{*}$ There was a significant difference compared with high fat model group, $p<0.05$. "There was a significant difference compared with control group, $p<0.05$. ${ }^{\Delta}$ There was a significant difference compared with high fat model group, $p<0.01$. \&There was a significant difference compared with low concentration Lactobacillus group, $p<0.05$. Al - TC/HDL-C. Group A - human low group, Group B - human high group, Group C - mice low group, Group D - mice high group, Group E - plant low group, Group F - plant high group, Group G Xuezhikang group, Group H - diet group, Group I - high fat group, Group I - control group.

Table III. Four weeks of treatment, blood biochemical index results $(\bar{x} \pm s, n=5)$

\begin{tabular}{|c|c|c|c|c|c|c|c|c|}
\hline $\begin{array}{l}\text { Project/ } \\
\text { groups }\end{array}$ & $\begin{array}{c}\mathrm{TC} \\
(\mathrm{mmol} / \mathrm{l})\end{array}$ & $\begin{array}{c}\mathrm{TG} \\
(\mathrm{mmol} / \mathrm{l})\end{array}$ & $\begin{array}{c}\mathrm{HDL} \\
(\mathrm{mmol} / \mathrm{l})\end{array}$ & $\begin{array}{c}\text { LDL } \\
(\mathrm{mmol} / \mathrm{l})\end{array}$ & $\begin{array}{c}\text { ALT } \\
(\mathrm{U} / \mathrm{I})\end{array}$ & $\begin{array}{l}\text { AST } \\
(\mathrm{U} / \mathrm{l})\end{array}$ & Al & $\begin{array}{c}\text { Glu } \\
(\mathrm{mmol} / \mathrm{l})\end{array}$ \\
\hline Group A & $1.34 \pm 0.42^{*}$ & $2.82 \pm 0.79^{\Delta}$ & $0.49 \pm 0.11^{\Delta}$ & $0.19 \pm 0.07^{\Delta}$ & $25.30 \pm 6.00^{\Delta}$ & $83.30 \pm 3.90$ & $1.68 \pm 0.42^{\# \Delta}$ & $6.96 \pm 0.63$ \\
\hline Group B & $1.38 \pm 0.36^{\star \#}$ & $2.48 \pm 0.30^{\Delta}$ & $0.45 \pm 0.04^{\Delta}$ & $0.16 \pm 0.02^{\Delta}$ & $29.00 \pm 4.10^{\Delta}$ & $85.40 \pm 19.80$ & $2.05 \pm 0.70^{\# \Delta}$ & $7.94 \pm 0.69$ \\
\hline Group C & $0.77 \pm 0.39^{*}$ & $2.25 \pm 0.34^{*}$ & $0.53 \pm 0.13^{*}$ & $0.20 \pm 0.09^{*}$ & $26.50 \pm 7.70^{\Delta}$ & $98.30 \pm 28.30$ & $0.61 \pm 1.02^{\Delta}$ & $7.45 \pm 0.18$ \\
\hline Group E & $1.53 \pm 0.70^{*}$ & $2.78 \pm 0.56^{\Delta}$ & $0.48 \pm 0.03^{\Delta}$ & $0.19 \pm 0.05^{\# \Delta}$ & $28.00 \pm 4.30^{\Delta}$ & $86.40 \pm 12.80$ & $1.57 \pm 0.69^{\Delta}$ & $6.50 \pm 1.07$ \\
\hline Group F & $0.96 \pm 0.17^{\Delta}$ & $2.63 \pm 0.45^{\Delta}$ & $0.47 \pm 0.07^{\Delta}$ & $0.16 \pm 0.03^{\Delta}$ & $29.60 \pm 4.00^{\Delta}$ & $96.60 \pm 13.60$ & $1.06 \pm 0.36^{\Delta}$ & $8.08 \pm 0.85$ \\
\hline Group G & $1.11 \pm 0.42^{\star}$ & $2.70 \pm 0.63^{\Delta}$ & $0.47 \pm 0.09^{\star \Delta}$ & $0.18 \pm 0.05^{\Delta}$ & $25.40 \pm 5.70^{\Delta}$ & $87.80 \pm 20.40$ & $1.42 \pm 1.14^{\Delta}$ & $7.52 \pm 1.02$ \\
\hline Group J & $0.89 \pm 0.27^{\Delta}$ & $2.68 \pm 0.43^{\Delta}$ & $0.48 \pm 0.05^{\Delta}$ & $0.21 \pm 0.02^{\Delta}$ & $25.50 \pm 7.00^{\Delta}$ & $84.20 \pm 16.70$ & $0.84 \pm 0.42^{\Delta}$ & $6.48 \pm 1.59$ \\
\hline
\end{tabular}

${ }^{*}$ There was a significant difference compared with high fat model group, $p<0.05$. "There was a significant difference compared with control group, $p<0.05 .{ }^{\Delta}$ There was a significant difference compared with high fat model group, $p<0.01$.

in the hyperlipidemic group $(p<0.05)$, while the level of TC was lower in the plant high group than in the control group $(p<0.05)$. In the drug treatment group, the levels of TG, HDL-C and LDL-C were lower than those in the hyperlipidemic group $(p<0.05)$, while the level of TC was lower than in the control group $(p<0.05)$.

\section{Serum biochemical indicators after four weeks of treatment}

The results of the serum biochemical indicators of mice in each group after four weeks of treatment are presented in Table III.
In the human low group, the levels of TC, TG, HDL-C, LDL-C and ALT, and the atherosclerosis index (AI) were lower than those in the hyperlipidemic group ( $p<0.05)$, while the Al was lower than in the control group $(p<0.05)$. In the human high group, the levels of TC, TG, HDL-C, LDL-C and ALT, and the Al were lower than those in the hyperlipidemic group $(p<0.05)$, while the $\mathrm{Al}$ and TC were lower than those in the control group $(p<0.05)$. In the mouse low group, the levels of TC, TG, HDL-C, LDL-C and ALT, and the $\mathrm{Al}$ were lower than those in the hyperlipidemic group $(p<0.05)$. In the mouse high group, the levels of TC, TG, HDL-C, LDL-C and ALT, and 


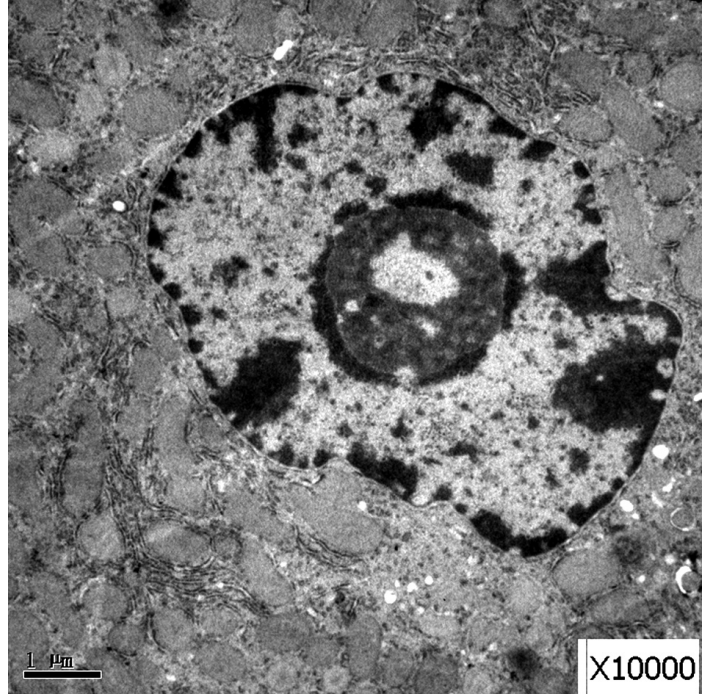

Figure 1. Liver sections of mice in the hyperlipidemic models at $10,000 \times$

the Al were lower than those in the hyperlipidemic group ( $p<0.05)$, while LDL was lower than that in the control group $(p<0.05)$. In the plant low group, the levels of TC, TG, HDL-C, LDL-C and ALT, and the $\mathrm{Al}$ were lower than those in the hyperlipidemic group ( $p<0.05$ ), while LDL was lower than that in the control group $(p<0.05)$. In the plant high group, the levels of TC, TG, HDL-C, LDL-C and ALT, and the Al were lower than those in the hyperlipidemic group $(p<0.01)$. In the Xuezhikang treatment group, the levels of TC, TG, HDL-C, LDL-C and ALT, and the Al were lower than those in the hyperlipidemic group $(p<0.05)$.

Changes in obesity indexes in each group during the treatment period

After four weeks of Lactobacillus treatment, the serum cholesterol and lipid levels, and the Al in each treatment group were all lower than those the hyperlipidemic group, and the difference was statistically significant. However, when compared with those in mice fed with a normal diet in the control group, only mice in the mouse low group had an Al lower than the controls, and the difference was not statistically significant. The indexes in other treatment groups were all higher than those in the controls, and a significant difference only existed in TC in the human high group, and the $\mathrm{Al}$ in the human low and human high groups.

\section{Electron microscopy observation results}

Obvious lipid droplets were found under a light microscope when the liver of mice in the hyperlipidemic model group was observed (Figure 1). Under an electron microscope, nucleus pyknosis,

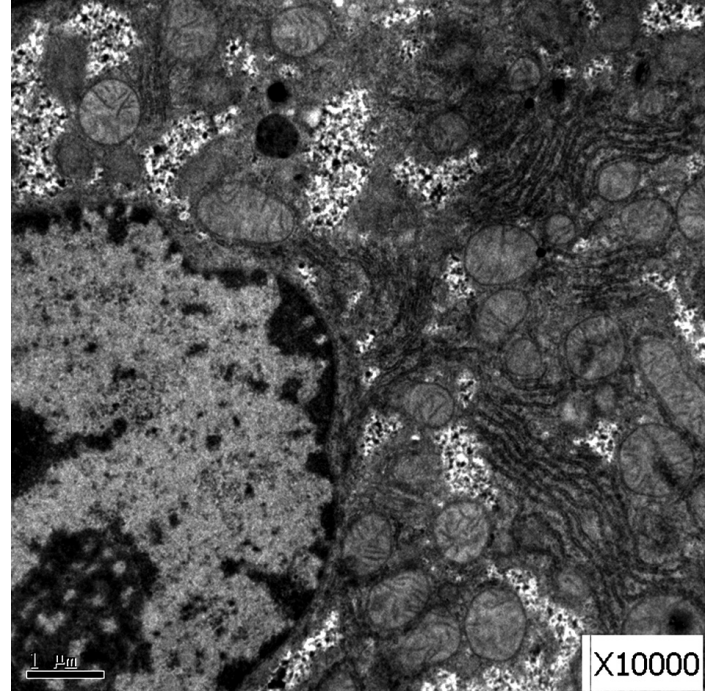

Figure 2. Liver sections of normal control mice at $10,000 \times$

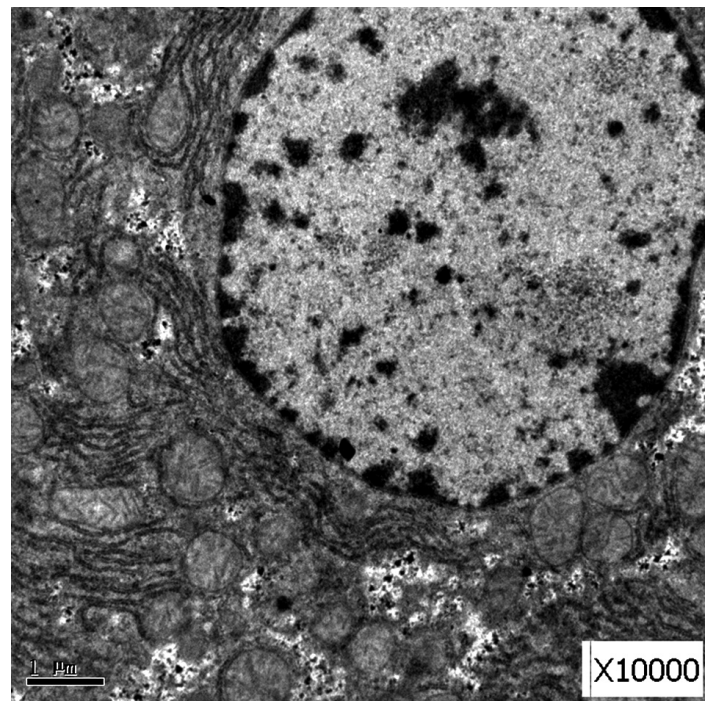

Figure 3. Liver sections of mice in the Lactobacillus treatment group at 10,000x

chromatin edge gathering, enlarged and rounded mitochondria, and blurred mitochondrial cristae could be observed. However, there was no abnormal structure of liver cells in the normal control group under a light and electron microscope (Figure 2). Furthermore, hepatic cells in the Lactobacillus treatment group were not abnormal under an electron microscope (Figure 3).

\section{Discussion}

Establishment of the hyperlipidemic mice model

It has been proven by experiments that the success rate of the dietary modeling of hyperlipidemic mice with $45 \%$ of total energy supplied by fat is the highest. This was due to the fact that a high- 
fat diet contains lard and cholesterol, which led to the increase in TC and TG. Furthermore, the highfat diet can increase the obesity index and blood lipid of mice, and subsequently lead to liver injury, and even fatty degeneration. In the present study, all mice were tested for blood biochemical indicators to prove the success of the model.

\section{Effects of Lactobacillus on hyperlipidemia}

Lactobacillus may transform cholesterol into fecal sterol through oxidation in the intestinal tract, which can no longer return to the liver through intestinal-hepatic circulation, thereby lowering cholesterol levels. In addition, there are special enzymes produced by Lactobacillus that can reduce cholesterol. These may inhibit the synthesis of cholesterol by inhibiting HMGCR (the rate-limiting enzymes in cholesterol synthesis). Lactobacillus bacteria also have antioxidant effects. The cellular and acellular extracts of Lactobacillus have different scavenging capacities for various free-radicals in vitro, which are closely correlated to the formation of atherosclerosis. In the present study, the lipid metabolism in hyperlipidemic mice improved at three weeks after Lactobacillus intragastric administration. TC, TG and LDL-C decreased in each group to different degrees. The longer the treatment duration was, the more obvious the improvement became. At the fourth week of treatment, these indexes were significantly lower than those in the "hyperlipidemic group", which is consistent with the results reported by Song [11]. However, in this study, the lipid-lowering effects of strains from different sources did not have a positive correlation with the concentration of the bacterial solution. In the study conducted by Huang et al., it was demonstrated that after adding Lr263, HDL-C and LDL-C changed in a dose-dependent manner. In addition, their research revealed that HDL-C exhibited different changes, with an increase in most cases, while TC, TG and LDL-C decreased [12]. However, in the present study, the HDL-C levels decreased, which is inconsistent with the previous results. What led to these results - the difference in strains, the difference in concentration, or the different durations of treatment? The longest duration of treatment in the present study was four weeks, while the duration for Huang's treatment was six weeks. In Kim's study, probiotics containing Lactobacillus were used to treat mice for eight weeks, and this also exhibited an increase in HDL-C [13]. Costabile et al. conducted a similar study in a population. Within 6-12 weeks of treatment, HDL-C increased by $14.7 \%$ [14] in subjects older than 60 years. Thus, it appears that treatment duration is a very important factor. At present, the commonly used drugs for treating hyperlipidemia are simvastatin, pravastatin, fluvastatin and Xuezhikang. These drugs can reduce intracellular LDL-C synthesis and plasma LDL-C concentration mainly by inhibiting the rate-limiting enzyme (HMG-CoA reductase) in the early stage of intracellular cholesterol synthesis. However, adverse reactions such as rhabdomyolysis, nervous system damage and other side effects exist, and serious liver damage can also be caused. Lactobacillus are probiotic bacteria with good safety, and there is no significant therapeutic difference between drug treatments. This can be used as an effective method to treat hyperlipidemia. Furthermore, in addition to Lactobacillus and drug treatment, the hyperlipidemia index in the treatment group that switched to a normal diet after modeling also improved. Hence, it can be concluded that improving the diet can also play a role in reducing blood lipids, and that this is the first recommended method for reducing weight and fat content.

\section{Effects of Lactobacillus in the liver}

Further development of obesity and hyperlipidemia can also cause hepatic injury, leading to non-alcoholic steatohepatitis, and even hepatic cirrhosis [15]. In the present study, the livers of hyperlipidemic model mice exhibited obvious morphological changes, accompanied by abnormal function with elevated AST and ALT, which is also evidence of hepatic injury caused by a high-fat diet. At the third week after treatment, the serum levels of AST and ALT did not decrease in each group, but at the fourth week after treatment, the levels of ALT in each group were significantly lower than those in the hyperlipidemic group, and there were significant differences in the statistical analysis. Although AST did not return to the normal range, the results of the hepatic histological examination revealed that the hepatic fatty degeneration improved, which is consistent with the results reported by $\mathrm{Oh}$ and $\operatorname{Kim}[15,16]$. Studies have shown that Lactobacillus can improve hypercholesterolemia in mice by activating Lxr- $\alpha$ in the liver and intestine, thereby enhancing the excretion of fecal cholesterol and bile acid in the small intestine [17]. As a probiotic, in addition to reducing blood lipid, it also has antioxidant and anti-inflammatory effects, which can improve the pathology in chronic low-grade inflammation related to obesity or metabolic syndrome $[18,19]$. This is also one of the reasons for the improvement in hepatic function.

\section{Protective effects of Lactobacillus for the heart}

Elevated lipid levels have been widely recognized as major risk factors for atherosclerosis, 
coronary heart disease and other cardiovascular disease (CVD). Increasing evidence suggests that intestinal disorders caused by a high-fat diet may promote the development of hyperlipidemia, obesity, insulin resistance and other metabolic syndromes. Studies have shown that probiotics, such as Lactobacillus, can reduce the $\mathrm{Al}$ (TC/HDL-C) [20] in mice fed with a high-fat diet, and cause the lag time of LDL-C oxidation to significantly improve, which reflect its antioxidant and cardioprotective properties [21]. In the present study, the Al in each treatment group was approximately 4 at the third week after treatment, and there was no difference between the hyperlipidemic model group and treatment group. However, the Al in each treatment group was significantly lower compared to the model group, at the fourth week of treatment, and the difference was statistically significant. This further confirms the cardioprotective effect of Lactobacillus and its positive correlation with the duration of treatment.

\section{Different strains of Lactobacillus in clinical practice}

Members of the genus Lactobacillus have long been recognized as being among the most abundant microorganisms in the human gastrointestinal tract and are associated with good intestinal health. These bacteria are often positively or negatively correlated with human diseases and chronic conditions. Therefore, the importance of this genus to human health cannot be ignored.

The most abundant Lactobacillus included $L$. delbrueckii, L. murinus, L. casei, L. rhamnosus, L. plantarum, and $L$. ruminis. Some of these species (e.g. $L$. rhamnosus and L. murinus) are rarely isolated from the parenteral environment and are considered to be gut-autochthonous microorganisms. Other mucosal sites are colonized by distinct species (e.g. L. crispatus in the vagina) [22]. There is also host specificity in some Lactobacillus species, e.g. L. reuteri [23].

Autochthonous Lactobacillus might play a role in the resolution of infectious disease and restoration of immune homeostasis; for instance, oral gavage with $L$. reuteri reduced anti-commensal antibodies, innate cytokines, and Th17 responses, thereby ameliorating immune hyper-reactivity [24]. The intestinal microbiota of patients with severe and early onset rheumatoid arthritis (RA) were shown to have increased proportions of $L$. salivarius, L. ruminis, and $L$. iners when compared to healthy, age-matched individuals [25]. There are also reports on the association of intestinal Lactobacillus with obesity in humans; e.g. lean women presented higher concentrations of $L$. plantarum compared to obese women [26]. Also, increased levels of $L$. gasseri were found in patients with type 2 diabetes [27]. In addition, the development of Lactobacillus occurs primarily during infancy, and growing evidence has emphasized its positive role and implications for human health, such as improving functional gastrointestinal disorders and allergies [28].

In conclusion, in this study we found that Lactobacillus can improve the high cholesterol, high blood lipid, and injury of hepatic function, and prevent further development of atherosclerosis caused by a high-fat diet to some extent. Correct dietary structure is the basis for the treatment of dietary hyperlipidemia and its complications. Lactobacillus and other probiotics can be used as an alternative or supplement to the existing treatment of non-alcoholic fatty liver disease, in order to reduce the risk of CVD.

\section{Acknowledgments}

This study was supported by the Zhejiang Science and Technology Bureau Public Welfare Project (No. LGF18H 180012) and Wenzhou Science and Technology Bureau (No. 2017Y1028).

\section{Conflicts of interest}

The authors declare no conflict of interest.

\section{References}

1. Wang X, Li M, Li X, Li Q. The analysis of the trend of serum lipid levels of Chinese residents. J. Taishan Med Colg 2010; 31: 106-8.

2. Wu HM, Tian Y, Yu JQ, Zhang R, Zhang XP, Guo P. The pandanus tectorius fruit extract (PTF) modulates the gut microbiota and exerts anti-hyperlipidaemic effects. Phytomedicine 2019; 58: 152863.

3. Chen K, Li S, Chen F, Li J, Luo XG. Regulation of the Lactobacillus strains on HMGCoA reductase gene transcription in human HepG2 cells via nuclear factor-кB. J Microbiol Biotechnol 2016; 26: 402-7.

4. Lim FT, Lim SM, Ramasamy K. Pediococcus acidilactici LAB4 and Lactobacillus plantarum LAB12 assimilate cholesterol and modulate ABCA1, CD36, NPC1L1 and SCARB1 in vitro. Benef Microbes 2017; 8: 97-109.

5. Zavišić G, Ristić S, Petričević S, et al. Characterization and preliminary lipid-lowering evaluation of Lactobacillus isolated from a traditional Serbian dairy product. Benef Microbes 2015; 6: 119-28.

6. Wang JG. Screening of cholesterol-lowering probiotic Lactobacillus and its cholesterol-lowering effect. Huhhot: Inner Mongolia Agricultural University; 2008.

7. Chen DW, Guo FX, Gu RX, Zou DW, Zhao HQ, Zhai HX. Screening and functional study of human lactic acid bacteria for reducing blood lipid. Food Ferment Ind 2014; 40.

8. Ma JM, Gong WJ, Wu CY, Jin MC. Rapid determination of cholesterol in eggs by direct saponification-ammonium ferric sulfate colorimetry. Chin J Health Inspec 2001; 11.

9. Shao YX. Medical Laboratory Animal Science Course. $2^{\text {nd }}$ edition. Nanjing: Southeast University Press; 2009.

10. Zuo HH, Huang JF, Liu SY, et al. Effect of Xuezhikang preconditioning on Fas/FasL mRNA expression in myo- 
cardium of rats with acute ischemia-reperfusion in vivo. Jiangxi Med J 2008; 43: 126-30.

11. Song JJ, Tian WJ, Kwok LY, et al. Effects of microencapsulated Lactobacillus plantarum LIP-1 on the gut microbiota of hyperlipidaemic rats. Brit J Nutr 2017; 118: 481-92.

12. Huang WC, Chen YM, Kan NW, et al. Hypolipidemic effects and safety of Lactobacillus Reuteri 263 in a hamster model of hyperlipidemia. Nutrients 2015; 7: 3767-82.

13. Kim SJ, Park SH, Sin HS, et al. Hypocholesterolemic effects of probiotic mixture on diet-induced hypercholesterolemic rats. Nutrients 2017; 9: e293.

14. Costabile A, Buttarazzi I, Kolida S, et al. An in vivo assessment of the cholesterol-lowering efficacy of Lactobacillus plantarum ECGC 13110402 in normal to mildly hypercholesterolaemic adults. PLoS One 2017; 12: e0187964.

15. Kim DH, Jeong D, Kang IB, Kim H, Song KY, Seo KH. Dual function of Lactobacillus kefiri DH5 in preventing highfat-diet-induced obesity: direct reduction of cholesterol and upregulation of PPAR- in adipose tissue. Mol Nutr Food Res 2017; 61: 10.

16. Oh NS, Koh JH, Park MR, Kim Y, Kim SH. Short communication: hypolipidemic and antiinflammatory effects of fermented Maillard reaction products by Lactobacillus fermentum H9 in an animal model. J Dairy Sci 2016; 99: 9415-23.

17. Heo W, Lee ES, Cho HT, et al. Lactobacillus plantarum LRCC 5273 isolated from Kimchi ameliorates diet-induced hypercholesterolemia in C57BL/6 mice. Biosci Biotechnol Biochem 2018; 82: 1964-72.

18. Park S, Ji Y, Jung HY, et al. Lactobacillus plantarum HACO1 regulates gut microbiota and adipose tissue accumulation in a diet-induced obesity murine model. Appl Microbiol Biotechnol 2017; 101: 1605-14.

19. Seddik HA, Bendali F, Gancel F, Fliss I, Spano G, Drider D. Lactobacillus plantarum and its probiotic and food potentialities. Probiotics Antimicro 2017; 9: 111-22.

20. Lye HS, Kato T, Low WY, et al. Lactobacillus fermentum FTDC 8312 combats hypercholesterolemia via alteration of gut microbiota. J Biotechnol 2017; 262: 75-83.

21. Chiu HF, Chen YJ, Lu YY, et al. Regulatory efficacy of fermented plant extract on the intestinal microflora and lipid profile in mildly hypercholesterolemic individuals. J Food Drug Anal 2017; 25: 819-27.

22. Gosmann C, Anahtar MN, Handley SA, et al. Lactobacillus-deficient cervicovaginal bacterial communities are associated with increased HIV acquisition in young South African women. Immunity 2017; 46: 29-37.

23. Duar RM, Frese SA, Fernando SC, et al. Experimental determination of host adaptation of Lactobacillus reuteri to different vertebrate species. Appl Environ Microbiol 2017; 83: e00132-17.

24. Kamdar K, Khakpour S, Chen J, et al. Genetic and metabolic signals during acute enteric bacterial infection alter the microbiota and drive progression to chronic inflammatory disease. Cell Host Microbe 2016; 19: 21-31.

25. Zhang $X$, Zhang D, Jia $H$, et al. The oral and gut microbiomes are perturbed in rheumatoid arthritis and partly normalized after treatment. Nat Med 2015; 21: 895-905.

26. Teixeira TFS, Grześkowiak LM, Salminen S, Laitinen K, Bressan J, Gouveia Peluzio Mdo C. Faecal levels of Bifidobacterium and Clostridium coccoides but not plasma lipopolysaccharide are inversely related to insulin and HOMA index in women. Clin Nutr 2013; 32: 1017-22.

27. Karlsson FH, Tremaroli V, Nookaew I, et al. Gut metagenome in European women with normal, impaired and diabetic glucose control. Nature 2013; 498: 99-103.
28. Goulet O, Hojsak I, Kolacek S, et al. Paediatricians play a key role in preventing early harmful events that could permanently influence the development of the gut microbiota in childhood. Acta Paediatr 2019; 108: 1942-54. 